\title{
Holographic Central Charge for 2-Dimensional de Sitter Space
}

\author{
by \\ A.J.M. Medved \\ Department of Physics and Theoretical Physics Institute \\ University of Alberta \\ Edmonton, Canada T6G-2J1 \\ [e-mail: amedved@phys.ualberta.ca]
}

\begin{abstract}
Recently, investigations have begun into a holographic duality for twodimensional de Sitter space. In this paper, we evaluate the associated central charge, using a modified version of the canonical Hamiltonian method that was first advocated by Catelani et al. Our computation agrees with that of a prior work (Cadoni et al), but we argue that the method used here is, perhaps, aesthetically preferable on holographic grounds. We also confirm an agreement between the Cardy and thermodynamic entropy, thus providing further support for the conjectured two-dimensional de Sitter/conformal field theory correspondence.
\end{abstract}




\section{Introduction}

To this date, the greatest triumph of the holographic principle [1, 2] remains the correspondence between anti-de Sitter (AdS) bulk spacetimes and conformal field theories (CFTs) of one dimension fewer $[3,4,5]$. On the basis of this success, there has been substantial interest in establishing a similar duality in a de Sitter (dS) context. There has indeed been undeniable progress in this direction [6, 7] (and, for instance, [8]-[14]), although unresolved issues still remain. (For a recent critique, see [15].)

For the most part, investigations into the (conjectured) dS/CFT correspondence have concentrated on three, four and five bulk dimensions; with three being the simplest case, and four and five being (presumably) the physically most relevant. Nonetheless, the intriguing case of two bulk dimensions has begun to draw some limited attention [16, 17]. These works ([17] in particular) have been significantly inspired by prior treatments on the $\mathrm{AdS}_{2} / \mathrm{CFT}_{1}$ correspondence (including [18]-[29]).

Naively, one might expect that holographic dualities in a two-dimensional bulk context would be the simplest cases of all. This may certainly be true on a calculational level; however, one finds such two-dimensional dualities to be plagued by conceptually ambiguous features. For instance, from a bulk perspective, it is conspicuously unclear as to how one should formulate a two-dimensional theory of gravity. This ambiguity is a direct consequence of the "conventional" Einstein tensor identically vanishing in two-dimensions of spacetime. The standard method of circumvention is to introduce another dynamical field into the mix; typically, an auxiliary scalar field known as the dilaton. However, there is generally no a priori rationale for modifying an otherwise pure gravitational theory in this manner. ${ }^{1}$ Furthermore, from the viewpoint of the conformal boundary theory, it is somewhat problematic that there is, formally speaking, no such entity as a one-dimensional conformal field theory. Hence, any notion of a straightforward (A) $\mathrm{dS}_{n} / \mathrm{CFT}_{n-1}$ correspondence is sabotaged when $n=2$. However, this last point is not quite as bleak as it sounds: a "CFT ${ }_{1}$ " can be feasibly interpreted as (for instance) a discrete light-cone quantization of a $\mathrm{CFT}_{2}$ [18] or a conformal-mechanical system coupled to an external source [28].

\footnotetext{
${ }^{1} \mathrm{~A}$ notable exception is when the two-dimensional theory is regarded as having a higherdimensional pedigree; in which case, the dilaton is usually associated with a compactified dimension.
} 
Let us now refocus our attention on the recent investigation into the $\mathrm{dS}_{2} / \mathrm{CFT}_{1}$ duality by Cadoni et al [17]. One of the highlights of this work was a calculation of the central charge associated with a holographically induced Virasoro algebra. (Significantly, the Virasoro algebra effectively describes the two-dimensional conformal group [30].) To this end, the authors employed a canonical Hamiltonian framework [31, 32]. More specifically, they deduced the central charge by considering the deformation algebra of the symmetry generators associated with the Hamiltonian asymptotic-boundary terms [33]. One technical caveat however: to obtain the "correct" the central charge (i.e., the value for which the Cardy formula [34] agrees with the thermodynamic entropy), it was also necessary to include the contribution from an inner boundary of the spacetime. (Notably, the same adjustment was used in the analogous $\mathrm{AdS}_{2} / \mathrm{CFT}_{1}$ calculation [28].) Although this is a technically sound procedure, it is arguably in conflict with the very essence of the holographic principle. That is to say, one might expect that a single connected boundary (such as a "preferred screen" [35]) would be capable of encoding all of the relevant information about the bulk spacetime. If this were the case, the asymptotic boundary theory should be, by itself, sufficient to reproduce the correct value of the central charge.

With the above discussion in mind, let us now consider a prior work, specific to the $\mathrm{AdS}_{2} / \mathrm{CFT}_{1}$ correspondence, by Catelani, Vanzo and Caldarelli (CVC) [27]. These authors advocated a "modified" canonical-Hamiltonian treatment as a means for resolving an apparent discrepancy between the statistical Cardy entropy and the thermodynamic entropy. (Historically speaking, the two entropies differed by a factor of $\sqrt{2}$ in the original $\mathrm{AdS}_{2} / \mathrm{CFT}_{1}$ calculation [19]. This discrepancy was later resolved by several distinct means: sacrificing diffeomorphism invariance [24], the forementioned inclusion of the inner-boundary contribution [28], and the method currently under consideration.) More to the point, CVC have argued that one should rigorously keep track of the various boundary terms that arise while formulating the canonical Hamiltonian. Note that this philosophy is contrary to the conventional practice of ignoring the boundary terms until they are explicitly needed. (In principle, one can retrieve the discarded terms by enforcing that the bulk Hamiltonian is differentiable. See, for instance, [36].)

As it turned out, the modifications suggested by CVC did indeed lead to a precise agreement between the statistical Cardy entropy and the $\mathrm{AdS}_{2}$ thermodynamic entropy. Moreover and significantly to our prior discussion, 
the central charge, as calculated by the CVC method, is strictly accounted for by the symmetry properties of a single (asymptotic) boundary. The purpose of the current paper is to see if the same success can be achieved in a two-dimensional de Sitter context.

The remainder of the paper is organized as follows. In Section 2, we introduce the relevant $\mathrm{dS}_{2}$ formalism, with the focus being on asymptotic symmetries. In Section 3, by way of a "modified" Hamiltonian framework (as discussed above), we calculate the central charge of the dually related CFT. Section 4 considers the Cardy statistical entropy. Finally, a brief summary is provided in Section 5.

\section{Preliminary Analysis}

In considering a two-dimensional theory of gravity, it is convenient to work with an explicit form for the action. Here, the most natural choice is the de Sitter analogue of Jackiw-Teitelboim gravity [37]. That is:

$$
I=\frac{1}{2} \int d^{2} x \sqrt{-g} \eta\left[R-\frac{2}{l^{2}}\right]
$$

where $\eta$ is the dilaton (scalar) field and $l$ is a fundamental parameter of dimension length. Let us take note of the field equations, which are obtained by varying the action with respect to the dilaton and metric (respectively):

$$
\begin{gathered}
R=\frac{2}{l^{2}}, \\
\square \eta=-\frac{2}{l^{2}} \eta .
\end{gathered}
$$

The former clearly indicates the desired property of a metric with a constant, positive curvature, whereas the latter usefully describes the dynamics of the dilaton.

To obtain the general, classical solution for the action (1), we can begin with the well-known Jackiw-Teitelboim solution [37]:

$$
d s_{J T}^{2}=-\left(\frac{r^{2}}{l^{2}}-a^{2}\right) d \tau^{2}+\left(\frac{r^{2}}{l^{2}}-a^{2}\right)^{-1} d r^{2}
$$




$$
\eta_{J T}=\eta_{0} \frac{r}{l}
$$

(where $a$ and $\eta_{0}$ are constants of integration) and then analytically continue from $l^{2}$ to $-l^{2}$. Along with an appropriate relabeling of the coordinates, this process yields:

$$
\begin{gathered}
d s^{2}=-\left(\frac{t^{2}}{l^{2}}+a^{2}\right)^{-1} d t^{2}+\left(\frac{t^{2}}{l^{2}}+a^{2}\right) d x^{2}, \\
\eta=\eta_{0} \frac{t}{l} .
\end{gathered}
$$

Note that we have also rescaled the dilaton field, which is permissible via the arbitrariness of $\eta_{0}$. (Also note that we will, without loss of generality, regard $\eta_{0}$ to be positive.)

At this point, some commentary on global properties is in order. In a strictly technical sense, the above solution covers the entire spacetime manifold $(-\infty \leq t, x \leq+\infty)$. However, it can be argued (for instance, [38]) that the surface of vanishing dilaton (in our case, at $t=0$ ) should act as a boundary of the spacetime. This argument follows from the observation that $\eta^{-1}$ effectively serves as the gravitational coupling "constant" in two-dimensional gravity theories. On this basis, we will view the manifold as being limited to non-negative values of $t$. Note that the asymptotic boundary at $t \rightarrow \infty$ complies with the usual (de Sitter) notion of spacelike future infinity or $\mathcal{I}^{+} .^{2}$

Although the spacetime is lacking a conventional event horizon (or any black hole-like structure), one can still anticipate thermodynamic properties in analogy to those associated with a black hole. This presumption follows from the existence of a surface, at $t=0$, behind which nothing can communicate with infinity. That is to say, such a surface naturally leads to an entanglement-like entropy and its associated thermodynamic structure. As in higher-dimensional de Sitter spacetimes, the absence of a globally time-like Killing vector complicates any attempt at rigorously calculating the thermodynamics in question. ${ }^{3}$ Nonetheless, one can still anticipate that the various thermodynamic properties are, at a classical level, essentially equivalent to

\footnotetext{
${ }^{2}$ Conversely, we could have, just as legitimately, limited the manifold to non-positive values of $t$; in which case, the asymptotic boundary would describe spacelike past infinity or $\mathcal{I}^{-}$.

${ }^{3}$ Although not fatally so. See, for instance, [11].
} 
those of a Jackiw-Teitelboim black hole. This follows from the two spacetimes being related by an almost trivial analytic continuation. ${ }^{4}$ Indeed, some quantitative analysis of the action (1) has already come out in support of this conjecture [17]. Thus, we can utilize the following thermodynamic identities (as measured by an observer at infinity) [39]:

$$
\begin{gathered}
T=\frac{|a|}{2 \pi l}, \\
|M|=\frac{a^{2} \eta_{0}}{2 l}, \\
S=2 \pi \eta_{o}|a|,
\end{gathered}
$$

with these quantities representing the classical values of temperature, conserved mass and entropy (respectively). Note the ambiguity in the sign of the conserved mass, which is an inherent feature of de Sitter space regardless of the dimensionality (see the "note added" in [8]).

Let us now extend considerations to spacetimes that are, in some sense, merely "asymptotically de Sitter". Closely following prior studies on (A) $\mathrm{dS}_{3}$ and $(\mathrm{A}) \mathrm{dS}_{2}$ (most recently, [17]), we will say that a solution is asymptotically de Sitter if it conforms to the following set of boundary conditions as $t \rightarrow \infty$ :

$$
\begin{gathered}
g_{x x}=\frac{t^{2}}{l^{2}}+\gamma_{x x}(x)+\mathcal{O}\left(t^{-2}\right), \\
g_{t t}=-\frac{l^{2}}{t^{2}}+\frac{l^{4}}{t^{4}} \gamma_{t t}(x)+\mathcal{O}\left(t^{-6}\right), \\
g_{t x}=\frac{l^{3}}{t^{3}} \gamma_{t x}(x)+\mathcal{O}\left(t^{-5}\right), \\
\eta=\eta_{0}\left[\frac{t}{l} \rho(x)+\frac{l}{t} \frac{\gamma_{\eta \eta}(x)}{2}+\mathcal{O}\left(t^{-3}\right)\right],
\end{gathered}
$$

where the $\gamma$ 's and $\rho$ are model-dependent functions. Note that $\rho \sim 1$ near the classical configuration.

\footnotetext{
${ }^{4}$ Such an argument has, of course, no validity when quantum corrections become important.
} 
The most general diffeomorphisms which preserve the above boundary conditions are found to be as follows: ${ }^{5}$

$$
\begin{gathered}
\xi^{x}[\epsilon]=\epsilon(x)+\frac{1}{2} \frac{l^{4}}{t^{2}} \epsilon^{\prime \prime}(x)+\mathcal{O}\left(t^{-4}\right), \\
\xi^{t}[\epsilon]=-t \epsilon^{\prime}(x)+\mathcal{O}\left(t^{-1}\right),
\end{gathered}
$$

where $\epsilon$ is an arbitrary function of $x$. Note that $\delta_{\epsilon} \eta=\mathcal{L}_{\epsilon} \eta \sim \mathcal{O}(t) \sim \mathcal{O}(\eta)$, which may seem severe, but is a necessary consequence of preserving the scalar nature of the dilaton (i.e., preserving diffeomorphism invariance of the action) [19]. Further note that, when $\epsilon=0$, the higher-order terms can be identified with pure gauge transformations [40].

It is straightforward to show that, up to gauge transformations, the boundary fields of Eqs.(11-14) transform as follows:

$$
\begin{gathered}
\delta_{\epsilon} \gamma_{t t}=\epsilon \gamma_{t t}^{\prime}+2 \epsilon^{\prime} \gamma_{t t}, \\
\delta_{\epsilon} \gamma_{x x}=\epsilon \gamma_{x x}^{\prime}+2 \epsilon^{\prime} \gamma_{x x}+l^{2} \epsilon^{\prime \prime \prime}, \\
\delta_{\epsilon} \gamma_{t x}=\epsilon \gamma_{t x}^{\prime}+3 \epsilon^{\prime} \gamma_{t x}-l \epsilon^{\prime \prime}\left(\gamma_{t t}+\gamma_{x x}\right), \\
\delta_{\epsilon} \rho=\epsilon \rho^{\prime}-\epsilon^{\prime} \rho, \\
\delta_{\epsilon} \gamma_{\eta \eta}=\epsilon \gamma_{\eta \eta}^{\prime}+\epsilon^{\prime} \gamma_{\eta \eta}+l^{2} \epsilon^{\prime \prime} \rho^{\prime} .
\end{gathered}
$$

It is a further point of interest that, if one sets $\epsilon=0$, and thus considers pure gauge transformations:

$$
\begin{aligned}
\xi^{x}[0] & =\frac{l^{4}}{t^{4}} \alpha^{x}(x), \\
\xi^{t}[0] & =\frac{l}{t} \alpha^{t}(x),
\end{aligned}
$$

than the theory is found to have (up to constant factors) only two gauge invariant quantities. These being $\rho(x)$ and:

$$
\theta(x) \propto \gamma_{x x}-\frac{1}{2} \gamma_{t t}
$$

\footnotetext{
${ }^{5}$ Here and throughout, a prime (dot) denotes a spatial (temporal) differentiation.
} 
As a consequence, we are always free to choose a gauge for which $\gamma_{t x}=0$. This luxury will prove to be convenient in later analysis.

Finally, it is worth noting that the dilatonic field equation (3), when translated to the solution of Eqs.(11-14), yields the relation:

$$
l^{2} \rho^{\prime \prime}=\rho\left(\gamma_{t t}-\gamma_{x x}\right)-\gamma_{\eta \eta} .
$$

This relation will prove to be another useful tool later on.

\section{Hamiltonian Formalism}

A canonical Hamiltonian framework [31, 32] has often been used, with considerable success, for studies on two-dimensional dilatonic gravity (for instance, [36]). In such studies, the prevailing wisdom has been that the various boundary terms (which arise whenever one differentiates by parts) can be harmlessly ignored until later in the calculation. Significantly to this approach, one can (in principle) ultimately retrieve the discarded surface terms by imposing differentiability on the bulk Hamiltonian. On the other hand, Catelani et al have recently argued [27] that, to calculate the central charge of the conformal boundary theory, one should pedantically keep track of all boundary terms throughout the process. In the analysis to follow, we will endeavor to extend this latter philosophy to the $\mathrm{dS}_{2}$ model of the prior section.

Let us begin here by employing an ADM-like decomposition of the metric, as appropriate for a spacelike boundary:

$$
d s^{2}=N^{2} d x^{2}-\sigma^{2}(d t+V d x)^{2},
$$

where $N, \sigma$ and $V$ are gauge-dependent functions of the spacetime coordinates. With this parametrization, the action of Eq.(1) takes the form:

$$
I=I_{b u l k}+I_{\infty},
$$

where:

$$
I_{b u l k}=\int d t d x\left[\frac{\eta^{\prime}}{N}\left(\sigma^{\prime}-\dot{V} \sigma-V \dot{\sigma}\right)+\frac{\dot{\eta} V}{N}\left(\dot{V} \sigma+V \dot{\sigma}-\sigma^{\prime}\right)-\frac{\dot{\eta} \dot{N}}{\sigma}-\frac{\eta}{l^{2}} N \sigma\right]
$$




$$
I_{\infty}=\int d x\left[\frac{\eta \dot{N}}{\sigma}+\frac{\eta V}{N}\left(\sigma^{\prime}-\dot{V} \sigma-V \dot{\sigma}\right)\right]_{\mathcal{I}^{+}} .
$$

Note that we have, in the prescribed manner, explicitly included any surface term arising at spacelike future infinity $\left(\mathcal{I}^{+}\right)$. Strictly speaking, there are also surface terms associated with the inner boundary, and these will play an important role in constraining the geometry near $t=0$. However, such terms should have no influence on the action of the asymptotic conformal group [27] and, hence, will be left out of the current discussion.

Varying the total action (27) with respect to small deformations in the four fields $(\eta, \sigma, N, V)$, we obtain a series of variational terms at $\mathcal{I}^{+}$. We are able to eliminate many of these terms by sensibly imposing that the geometry is fixed at the asymptotic boundary; that is, $\delta \eta=\delta \sigma=\delta N=\delta V=0$ at $\mathcal{I}^{+}$. After which, the following terms still remain:

$$
\int d x\left[\frac{\eta}{\sigma} \delta \dot{N}+\frac{\eta V}{N}\left(\delta \sigma^{\prime}-\sigma \delta \dot{V}-V \delta \dot{\sigma}\right)\right]_{\mathcal{I}^{+}} .
$$

It is now evident that, for a well-defined variational principle, the action should be supplemented by a surface contribution whose variation precisely cancels Eq.(30). Denoting this supplementary term as $\tilde{I}_{\infty}$, we have:

$$
\tilde{I}_{\infty}=-\int d x\left[\frac{\eta \dot{N}}{\sigma}+\frac{\eta V}{N}\left(\sigma^{\prime}-\dot{V} \sigma-V \dot{\sigma}\right)\right]_{\mathcal{I}^{+}} .
$$

Ignoring the boundary supplement $\left(\tilde{I}_{\infty}\right)$ for just a moment, we know that the original action (27) gives rise to the following canonical Hamiltonian up to surface terms: ${ }^{6}$

$$
H_{b u l k}=\int d t\left[\Pi_{\eta} \eta^{\prime}+\Pi_{\sigma} \sigma^{\prime}+\Pi_{N} N^{\prime}+\Pi_{V} V^{\prime}-\mathcal{L}\right],
$$

where the Lagrangian $(\mathcal{L})$ and canonical momenta $\left(\Pi_{\psi}\right)$ will be defined in the usual manner. That is:

$$
\int d^{2} x \mathcal{L}=I_{b u l k},
$$

\footnotetext{
${ }^{6}$ Here, we are following the standard Dirac program [31], except that time and position have exchanged their usual roles.
} 


$$
\begin{gathered}
\Pi_{\eta}=\frac{\delta \mathcal{L}}{\delta \eta^{\prime}}=\frac{1}{N}\left[\sigma^{\prime}-\dot{V} \sigma-V \dot{\sigma}\right], \\
\Pi_{\sigma}=\frac{\delta \mathcal{L}}{\delta \sigma^{\prime}}=\frac{1}{N}\left[\eta^{\prime}-\dot{\eta} V\right], \\
\Pi_{N}=\frac{\delta \mathcal{L}}{\delta N^{\prime}}=0 \\
\Pi_{V}=\frac{\delta \mathcal{L}}{\delta V^{\prime}}=0 .
\end{gathered}
$$

Some straightforward calculation verifies that $H_{b u l k}$ can be expressed as a linear combination of "weakly vanishing" (in a Dirac sense [31]) constraints. In particular, one finds the following up to surface terms:

$$
H_{\text {bulk }}=\int d t\left[V \mathcal{H}_{V}+N \mathcal{H}_{N}\right]
$$

where:

$$
\begin{gathered}
\mathcal{H}_{V}=\dot{\eta} \Pi_{\eta}-\sigma \dot{\Pi}_{\sigma} \approx 0 \\
\mathcal{H}_{N}=\Pi_{\eta} \Pi_{\sigma}-\frac{\ddot{\eta}}{\sigma}+\frac{\dot{\eta} \dot{\sigma}}{\sigma^{2}}+\frac{1}{l^{2}} \eta \sigma \approx 0 .
\end{gathered}
$$

Note, however, that the transformation from Eq.(32) to Eq.(38) gives rise to the following asymptotic surface terms (which we collectively denote as $\tilde{H}_{\infty}$ ):

$$
\tilde{H}_{\infty}=-\left[\eta^{\prime} \frac{V}{N} \sigma+\dot{\eta}\left(\frac{N}{\sigma}-\frac{V^{2} \sigma}{N}\right)\right]_{\mathcal{I}^{+}} .
$$

Let us re-emphasize that the bulk Hamiltonian has been formulated to vanish on the constraint surface. At this point, one normally considers (for instance, [36]) a total Hamiltonian of the form: $H=H_{b u l k}+J$, where $J$ is a surface contribution that ensures the differentiability of $H$ (i.e., ensures $\delta H=0$ on the relevant boundaries). Alternatively, we will follow Catelani et al [27] and consider:

$$
H=H_{b u l k}+J+K,
$$

where $J$ is still defined as above; whereas $K$ is a surface contribution that accounts for both $\tilde{H}_{\infty}(41)$ and the "supplementary" action term $\tilde{I}_{\infty}(31)$. More precisely, let us first define $\int d x \tilde{\mathcal{L}}_{\infty} \equiv \tilde{I}_{\infty}$ and then:

$$
\begin{aligned}
K & \equiv \tilde{H}_{\infty}-\tilde{\mathcal{L}}_{\infty} \\
& =\left[\frac{1}{\sigma}(\eta \dot{N}-\dot{\eta} N)+V\left(\eta \Pi_{\eta}-\sigma \Pi_{\sigma}\right)\right]_{\mathcal{I}^{+}}
\end{aligned}
$$


where Eqs. $(34,35)$ have been used to simplify the lower line.

Let us now see what we can learn about asymptotic symmetries in this Hamiltonian framework. Inasmuch as $H_{b u l k} \approx 0$, it is, for our purposes, sufficient to consider the action of the surface terms, $J$ and $K$. Beginning with $J$ and employing the above prescription, we have:

$$
\begin{aligned}
\delta J & =-\left.\delta H_{b u l k}\right|_{\mathcal{I}^{+}} \\
& =\left[\frac{N}{\sigma}\left(\delta \dot{\eta}-\frac{\dot{\eta}}{\sigma} \delta \sigma\right)-\frac{\dot{N}}{\sigma} \delta \eta+V\left(\sigma \delta \Pi_{\sigma}-\Pi_{\eta} \delta \eta\right)\right]_{\mathcal{I}^{+}} .
\end{aligned}
$$

An underlying premise of the canonical Hamiltonian framework is that a given symmetry generator, $\xi^{\mu}[\epsilon]$, has an associated conserved charge, $J[\epsilon]$, which can be expressed as (for instance, [19]):

$$
\delta J[\epsilon]=\left[\frac{\epsilon^{\perp}}{\sigma}\left(\delta \dot{\eta}-\frac{\dot{\eta}}{\sigma} \delta \sigma\right)-\frac{\dot{\epsilon}^{\perp}}{\sigma} \delta \eta+\epsilon^{\|}\left(\sigma \delta \Pi_{\sigma}-\Pi_{\eta} \delta \eta\right)\right]_{\mathcal{I}^{+}},
$$

where $\epsilon^{\perp}=N \epsilon^{x}$ and $\epsilon^{\|}=\epsilon^{t}+V \epsilon^{x}$.

For the purpose of evaluating Eq.(45), let us now reconsider Eqs.(11-14) for the asymptotic form of the metric. We can further fix the gauge, without loss of generality, ${ }^{7}$ so that $\gamma_{t x}=0$. Comparing with Eq.(26), we then obtain $V=0$ and the following relations:

$$
\begin{gathered}
N=\frac{t}{l}+\frac{l}{t} \frac{\gamma_{x x}(x)}{2}+\mathcal{O}\left(t^{-3}\right) \\
\sigma=\frac{l}{t}-\frac{l^{3}}{t^{3}} \frac{\gamma_{t t}(x)}{2}+\mathcal{O}\left(t^{-5}\right), \\
\eta=\eta_{0}\left[\frac{t}{l} \rho(x)+\frac{l}{t} \frac{\gamma_{\eta \eta}(x)}{2}\right]+\mathcal{O}\left(t^{-3}\right) .
\end{gathered}
$$

Directly incorporating the above formalism into Eq.(45), we find:

$$
\delta J[\epsilon]=\eta_{0}\left[\frac{\epsilon}{l}\left(\gamma_{x x} \delta \rho+\frac{\rho}{2} \delta \gamma_{t t}-\delta \gamma_{\eta \eta}\right)-l \epsilon^{\prime} \delta \rho^{\prime}+l \epsilon^{\prime \prime} \delta \rho\right]
$$

where only terms that are finite as $t \rightarrow \infty$ have been retained.

\footnotetext{
${ }^{7}$ See the discussion immediately following Eq.(24).
} 
Our next objective is to obtain a similar expression for the remaining surface contribution, $K$ (43). In analogous fashion, the associated conserved charge, $K[\epsilon]$, takes the form:

$$
K[\epsilon]=\left[\frac{1}{\sigma}\left(\dot{\epsilon}^{\perp} \eta-\epsilon^{\perp} \dot{\eta}\right)+\epsilon^{\|}\left(\eta \Pi_{\eta}-\sigma \Pi_{\sigma}\right)\right]_{\mathcal{I}^{+}} .
$$

Reapplying Eqs.(46-48), the gauge choice $V=0$ and the $t \rightarrow \infty$ limit, we have:

$$
K[\epsilon]=\eta_{0}\left[\frac{\epsilon}{l}\left(\gamma_{\eta \eta}-\rho \gamma_{x x}\right)+l \epsilon^{\prime} \rho^{\prime}-l \epsilon^{\prime \prime} \rho\right] .
$$

Let us now define a "total" conserved charge: $\Psi[\epsilon] \equiv J[\epsilon]+K[\epsilon]$. Summing Eq.(45) and the variation of Eq.(51), we then obtain:

$$
\delta \Psi[\epsilon]=\eta_{0} \frac{\epsilon \rho}{2 l}\left[\delta \gamma_{t t}-2 \delta \gamma_{x x}\right]
$$

It is a point of interest, and certainly no coincidence, that the conserved charge can be expressed strictly in terms of gauge invariant quantities: $\rho$ and $\theta$ of Eq.(24).

Next, we will focus on configurations near the classical solution; that is, $\rho(x)=1+\bar{\rho}(x)$, where $\bar{\rho}(x)$ and the $\gamma^{\prime}$ s are much less than 1 . Some manipulation of Eq.(52) and application of the perturbative field equation (25) then leads to:

$$
\Psi[\epsilon]=l \eta_{0} \epsilon \bar{\rho}^{\prime \prime} \pm \epsilon M .
$$

Here, $M$ is an integration constant, which can be directly identified, up to a sign ambiguity, with the conserved mass of Eq.(9). This identity follows from the observation that, for a purely classical, on-shell solution, one should obtain $H[\epsilon]=\epsilon|M|$.

Applying the transformation equations (17-21) to Eq.(53) and defining $\epsilon \Theta \equiv \Psi[\epsilon]$, we find:

$$
\delta_{\omega} \Theta=\omega \Theta^{\prime}+2 \omega^{\prime} \Theta-l \eta_{0} \omega^{\prime \prime \prime}
$$

where Eq.(25) and the near-classical approximation have again been utilized.

Interestingly, although not unexpectedly, $\Theta$ transforms just as would the stress tensor of a conformal field theory [30]. This outcome can, at least conjecturally, be viewed as a manifestation of the dS/CFT correspondence $[6,9]$. That is, in analogy to the $\mathrm{AdS}_{2} / \mathrm{CFT}_{1}$ correspondence [18], the asymptotic 
symmetries of two-dimensional de Sitter gravity are expected to be a subgroup of the 1+1-dimensional conformal group. The third term in Eq.(54) is the anticipated anomalous derivative term, which is related to the central charge $(c)$ of the associated Virasoro algebra [30]. On this basis, it is possible to identify $c / 12=\eta_{0}$.

We can make the above observations more explicit by first taking note of the following expression for the deformation algebra [33]:

$$
\epsilon \delta_{\omega} \Theta=\{\epsilon \Theta, \omega \Theta\}_{D B}
$$

where the subscript "DB" denotes the Dirac bracket formalism [31]. There are, however, subtle difficulties in directly applying this formalism to the case of a one-dimensional boundary [19]. Nonetheless, Cadoni and Mignemi [22] have suggested the following circumvention:

$$
\epsilon \delta_{\omega} \Theta=\{\epsilon \hat{\Theta}, \omega \Theta\}_{D B}
$$

where the hat symbol signifies that, for a timelike (spacelike) boundary, this is a time (position) averaged quantity.

By way of Eqs. $(54,56)$ and the following Fourier expansions:

$$
\begin{gathered}
\epsilon=l \sum_{m} a_{m} e^{i m x / l}, \\
\omega=l \sum_{n} a_{n} e^{i n x / l}, \\
\Theta=\frac{1}{l} \sum_{k} L_{k} e^{-i k x / l}, \\
\epsilon \hat{\Theta}=\frac{1}{l} \int_{0}^{l} d x \epsilon \Theta=\sum_{m} a_{m} L_{m},
\end{gathered}
$$

some straightforward calculation yields:

$$
\left[L_{m}, L_{n}\right]=(m-n) L_{m+n}+\frac{12 \eta_{0}}{12} m^{3} \delta_{m+n, 0} .
$$

That is to say, the asymptotic symmetries of $\mathrm{dS}_{2}$ are indeed realized by a Virasoro algebra ${ }^{8}$ with a classical central charge of precisely:

$$
c=12 \eta_{0}
$$

\footnotetext{
${ }^{8}$ The more conventional form of the Virasoro algebra, $\left[L_{m}, L_{n}\right]=(m-n) L_{m+n}+$ $\frac{c}{12} m\left(m^{2}-1\right) \delta_{m+n, 0}$, can be obtained with a trivial shift: $L_{0} \rightarrow L_{0}+\frac{1}{2} \eta_{0}$.
} 
Notably, this is the same value for the central charge as found, via a different methodology, ${ }^{9}$ by Cadoni et al [17].

\section{Cardy Entropy}

By way of [17], we can already anticipate that the central charge of Eq.(62) will yield a statistical entropy in agreement with the classical thermodynamic value (10). However, this coincidence provides an important consistency check on the formalism, so let us explicitly confirm that this is indeed the case.

The calculation in question utilizes the well-known Cardy formula [34], which relates the density of states (i.e., statistical entropy) of a $\mathrm{CFT}_{2}$ to the associated Virasoro algebra. This formula is obtained by way of some formal manipulations that exploit the modular and conformal invariance of the $\mathrm{CFT}_{2}$ partition function (for a recent derivation, see [41]). More specifically, one finds that:

$$
S_{C a r}=2 \pi \sqrt{\frac{c \mathrm{£}_{0}}{6}},
$$

where matters have been simplified with the (standard) assumptions that $L_{0}>>c$ and $L_{0} \sim 0$ for the ground state.

Before proceeding, let us point out that the Cardy formula is, in fact, commonly employed as an intermediate step in statistically motivated calculations of black hole entropy (for example, [40]). Generally speaking, one focuses on a relevant boundary (either a horizon or an asymptotic infinity) and then relates the boundary dynamics to an effective Virasoro algebra. (Such a relation is often possible because of the high degree of symmetry of black hole spacetimes.) Although this methodology has enjoyed substantial success, there are several caveats that suggest the Cardy formula should be applied (in this manner) with some degree of caution. Most notably (see [42] for a thorough discussion): (i) it is not apparent as to how an asymptotic boundary theory can be sensitive to the details of the interior geometry, whereas it is not evident that a black hole horizon (which is an observerdependent construct) can be regarded as boundary at all, (ii) it has not been

\footnotetext{
${ }^{9}$ Both methods do, however, employ a canonical Hamiltonian framework. See Section 1 for further discussion.
} 
rigorously established that classical symmetries can be used to determine the quantum properties of a system, (iii) although such calculations are naively statistical in nature, it is not at all clear as to what degrees of freedom are actually being counted by the Cardy formula. With regard to the last point, it has, however, been argued that, for the very special case of a two-dimensional (fundamental) theory, the physical (black hole) degrees of freedom are truly being counted [20].

Let us now consider the direct application of Eq.(63) to the current analysis. Of course, $c$ is known (62), but it is still necessary to evaluate $L_{0}$ in terms of the bulk solution. This is possible by way of Eq.(59), which implies the following Fourier transform:

$$
L_{n}=\int_{0}^{l} d x \Theta e^{i n x / l}
$$

Substituting for $\Theta=\Psi / \epsilon$ via Eq.(53), we have for the classical solution $(\bar{\rho}=0)$ :

$$
\begin{aligned}
L_{0} & =l|M| \\
& =\frac{\eta_{0} a^{2}}{2}
\end{aligned}
$$

where Eq.(9) has been used to obtain the lower line.

It is now straightforward to evaluate Eq.(63) for the statistical entropy:

$$
S_{C a r}=2 \pi \eta_{0}|a|,
$$

in perfect agreement with the (classical) thermodynamic entropy of Eq.(10).

\section{Conclusion}

In summary, we have calculated the central charge in a $\mathrm{dS}_{2} / \mathrm{CFT}_{1}$ context by applying a methodology that has been advocated by Catelani, Vanzo and Caldarelli [27]. Our outcome substantiates a recent, related calculation by Cadoni et al [17]. Although the two methods are both technically valid, we have argued that, from a holographic perspective, the current treatment is (perhaps) the aesthetically preferable one. 
We went on to confirm that, given this value of the central charge, Cardy's statistical entropy [34] is in precise agreement with the classical thermodynamic quantity. This coincidence provides further support for the conjectured $\mathrm{dS}_{2} / \mathrm{CFT}_{1}[16,17]$ correspondence. This relationship may, of course, be a lower-dimensional realization of a more general holographic duality. It is, however, a puzzlement that, given a dS bulk spacetime of arbitrary dimensionality, the dually related CFT is generally regarded as being a non-unitary theory [6]. As an immediate consequence, such a theory can not be expected, a priori, to conform with Cardy's entropic formula. (Consult [12] for further discussion.) In all likelihood, this oddity will have to be better understood before the dS/CFT duality can be "promoted" beyond the conjectural level.

\section{Acknowledgments}

The author would like to thank V.P. Frolov for helpful conversations.

\section{References}

[1] G. 't Hooft, "Dimensional Reduction in Quantum Gravity", grqc/9310026 (1993).

[2] L. Susskind, J. Math. Phys. 36, 6377 (1995) [hep-th/9409089].

[3] J.M. Maldacena, Adv. Theor. Math. Phys. 2, 231 (1998) [hepth/9711200].

[4] S.S. Gubser, I.R. Klebanov and A.M. Polyakov, Phys. Lett. B428, 105 (1998) [hep-th/9802109].

[5] E. Witten, Adv. Theor. Math. Phys. 2, 253 (1998) [hep-th/9802150].

[6] A. Strominger, JHEP 0110, 034 (2001) [hep-th/0106113].

[7] E. Witten, "Quantum Gravity in De Sitter Space", hep-th/0106109 (2001).

[8] D. Klemm, Nucl. Phys. B625, 295 (2002) [hep-th/0106247]. 
[9] M.Spradlin, A. Strominger and A. Volovich, "Les Houches Lectures on De Sitter Space", hep-th/0110007 (2001).

[10] B. McInnes, Nucl. Phys. B627, 311 (2002) [hep-th/0110062].

[11] V. Balasubramanian, J. de Boer and D. Minic, Phys. Rev. D65, 123508 (2002) [hep-th/0110108].

[12] R. Bousso, A. Maloney and A. Strominger, Phys. Rev. D65, 104039 (2002) [hep-th/0112218].

[13] M. Spradlin and A. Volovich, Phys. Rev. D65, 104037 (2002) [hepth/0112223].

[14] V. Balasubramanian, J. de Boer and D. Minic, "Exploring de Sitter Space and Holography", hep-th/0207245 (2002).

[15] L. Dyson, M. Kleban and L. Susskind, "Disturbing Implications of a Cosmological Constant", hep-th/0208013 (2002).

[16] S. Ness and G. Siopsis, Phys. Lett. B536, 315 (2002) [hep-th/0202096].

[17] M. Cadoni, P. Carta, M. Cavaglia and S. Mignemi, "Two-Dimensional dS/CFT Correspondence", hep-th/0205211 (2002).

[18] A. Strominger, JHEP 9901, 007 (1999) [hep-th/9809027].

[19] M. Cadoni and S. Mignemi, Phys. Rev. D59, 081501 (1999) [hepth/9810251].

[20] D.V. Fursaev, "A Note on Entanglement Entropy and Conformal Field Theory", hep-th/9811122 (1998).

[21] V. Frolov, D. Fursaev, J. Gegenberg and G. Kunstatter, Phys. Rev. D60, 024016 (1999) [hep-th/9901087].

[22] M. Cadoni and S. Mignemi, Nucl. Phys. B557, 165 (1999) [hepth/9902040]; Phys. Rev. D61, 088502 (2000) [hep-th/9910225]; Phys. Lett. B490, 131 (2000) [hep-th/0002256].

[23] M. Spradlin and A. Strominger, JHEP 9911, 021 (1999) [hepth/9904143]. 
[24] J. Navarro-Salas and P. Navarro, Nucl. Phys. B579, 250 (2000) [hepth/9910076].

[25] D.J. Navarro, J. Navarro-Salas and P. Navarro, Nucl. Phys. B580, 311 (2000) [hep-th/9911091].

[26] M. Cadoni and M. Cavaglia, Phys. Lett. B499, 315 (2001) [hepth/0005179]; Phys. Rev. D63, 084024 (2001) [hep-th/0008084].

[27] G. Catelani and L. Vanzo, "On the sqrt\{2\} Puzzle in $\mathrm{AdS}_{2} / \mathrm{CFT}_{1}$ ", hep-th/0009186 (2000); M. Caldarelli, G. Catelani and L. Vanzo, JHEP 0010, 005 (2000) [hep-th/0008058].

[28] M. Cadoni, P. Carta, D. Klemm and S. Mignemi, Phys. Rev. D63, 125021 (2001) [hep-th/0009185].

[29] M. Cadoni and P. Carta, Mod. Phys. Lett. A16, 171 (2001) [hepth/0102064]; Phys. Lett. B522, 126 (2001) [hep-th/0107234].

[30] See, for instance, P. Di Francesco, P. Mathieu and D. Senechal, Conformal Field Theory (Springer, New York) (1997).

[31] P.A.M. Dirac, Lectures on Quantum Mechanics (Yeshiva University Press, New York) (1964).

[32] A. Hanson, T. Regge and C. Teitelboim, Constrained Hamiltonian Systems (Accademia Nazionale dei Lincei, Roma) (1976).

[33] J.D. Brown and M. Henneaux, Comm. Math. Phys. 104, 207 (1986).

[34] J.L. Cardy, Nucl. Phys. B270, 317 (1986).

[35] R. Bousso, JHEP 9906, 028 (1999) [hep-th/9906022].

[36] D. Louis-Martinez, J. Gegenberg and G. Kunstatter, Phys. Lett. B321, 193 (1994) [gr-qc/9309018].

[37] R. Jackiw in Quantum Theory of Gravity, ed. S. Christensen (Hilger, Bristol) (1984), p.403; C. Teitelboim, ibid, p.327; R. Jackiw, Nucl. Phys. B252, 343 (1985). 
[38] M. Cadoni, Phys. Lett. B395, 10 (1997) [hep-th/9610201].

[39] J. Gegenberg, G. Kunstatter and D. Louis-Martinez, Phys. Rev. D51, 1781 (1995) [gr-qc/9408015].

[40] A. Strominger, JHEP 9802, 009 (1998) [hep-th/9712251].

[41] S. Carlip, Class. Quant. Grav. 15, 3609 (1998) [hep-th/9806026].

[42] S. Carlip, Nucl. Phys. Proc. Suppl. 88, 10 (2000) [gr-qc/9912118]. 